\title{
Correction to: A Novel Solution Method for Free Vibration Analysis of Functionally Graded Arbitrary Quadrilateral Plates with Hole
}

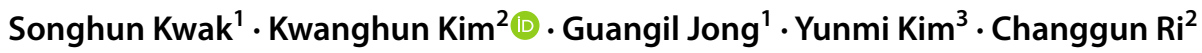

Published online: 13 June 2021

(c) Krishtel eMaging Solutions Private Limited 2021

Correction to: Journal of Vibration Engineering \& Technologies https://doi.org/10.1007/s42417-021-00327-5

The publication of this article unfortunately contained mistakes. The name of the country in affiliation was incorrect. Correct it should be "Democratic People's Republic of Korea".

The original article has been corrected.

1. College of Mechanical Science and Technology, Kim Chaek University of Technology, Pyongyang 950003, Democratic People's Republic of Korea

2. Department of Engineering Machine, Pyongyang University of Mechanical Engineering, Pyongyang 999093, Democratic People's Republic of Korea

The original article can be found online at https://doi.org/10.1007/ s42417-021-00327-5.

Kwanghun Kim

kimkwanghun@163.com

1 College of Mechanical Science and Technology, Kim Chaek University of Technology, Pyongyang 950003, Democratic People's Republic of Korea

2 Department of Engineering Machine, Pyongyang University of Mechanical Engineering, Pyongyang 999093, Democratic People's Republic of Korea

3 Department of Applied Mathematics, Kim Chaek University of Technology, Pyongyang 950003,

Democratic People's Republic of Korea
3. Department of Applied Mathematics, Kim Chaek University of Technology, Pyongyang 950,003, Democratic People's Republic of Korea

Publisher's Note Springer Nature remains neutral with regard to jurisdictional claims in published maps and institutional affiliations. 